\title{
An Innovative Solution to Enhance the Performance of Classical Heat Exchanger for Cooling Devices
}

\author{
Prashant Kumar $^{1}$, Kevin Schmidmayer ${ }^{1}$, Frederic Topin ${ }^{1}$, Marc Miscevic ${ }^{2}$ \\ ${ }^{1}$ IUSTI, CNRS UMR 7343, Aix-Marseille Université \\ 5, Rue Enrico Fermi, 13453 Marseille Cedex 13, France \\ prashant.kumar@univ-amu.fr; kevin.schmidmayer@univ-amu.fr; frederic.topin@univ-amu.fr \\ ${ }^{2}$ LAPLACE, CNRS UMR 5213, Université de Toulouse \\ 118, Route de Narbonne 31062 Toulouse Cedex 9, France \\ marc.mescevic@laplace.univ-tlse.fr
}

\begin{abstract}
The reliability and lifetime of many systems are very strongly related to the quality of the thermal management in many applications such as micro- and power- electronics or embedded systems. Recent technological advances lead to the development of miniaturised thermal systems. Improvement of thermo-hydraulic performance of such systems remains a great challenge. In this view, a future solution to enhance the overall performance of mini-channel heat exchanger at millimetre scale has been proposed to replace classical heat exchangers. It consists in dynamically deforming at least one of the walls in order to obtain a progressive corrugated channel. A systematic study on the different deformation parameters allowed obtaining the fluid flow and heat transfer characteristics of the system. 3-D numerical simulations were performed using commercial software based on the finite volume element in transient regime. It was observed that the dynamic deformation of the wall improves significantly the merit factor of the proposed system compared to straight and flat channel.
\end{abstract}

Keywords: heat transfer enhancement, wall morphing, dynamic deformation, merit factor, mini-channel heat exchanger

\section{Introduction}

The temperature control and associated heat flux management is crucial in many applications: micro -and powerelectronics, power station, air conditioners, heat pumps, chemistry, food, etc. The will to increase the performance and efficiency of these systems greatly amplifies this need as, in many situations; it becomes the limiting factor in optimizing system performance. Moreover, lifetime and reliability of many systems are very strongly related to the quality of the thermal management. Most structures today include a cooling system for extracting the heat dissipated during operation. This function must be performed throughout the operation but also act in a timely and fast way to meet sudden spikes. For example, the electrical power passes through an elementary component of the control electronics could be increased by a factor 10 or more (thus reducing the number of elementary components) if the performance of cooling system allowed evacuating the produced heat flux density.

During the last decades, significant changes in the design of power equipment have increased significantly the power density of embedded electronics. Hence the need to develop more advanced cooling systems and integrating new features. To improve heat transfer within a given system more intensified heat exchange solutions exist and are used. The majority of them are called "passive". They consist in most cases to increase and complicate the heat exchange surfaces. Notwithstanding the need to extract ever-greater powers and in some cases to specific moments brought us consider the use of techniques "active" intensification of heat transfer. These are implementing various physical mechanisms but have in common that an external mechanical power source is required for operation.

Add to this the fact that the main system mass is contained in the copper plate for the heat dissipation, the planar architecture of the electronics imposed by the cooling system and one understands the stakes in terms of compactness, performance and weight reduction.

It is nowadays common to meet chemical or thermal systems whose channels have submillimetric hydraulic diameters due to the increasing demands of new and effective mixing and heat transfer technologies for various industrial fields associated to the increasing need to control highly exothermic or explosive chemical reactions. If these systems can increase 
the compactness, one major drawback is the dififculty to disturb the boundary layers as lfow is mostly laminar in such smal devices and the net gain in terms of mass or heat transfer is due to the increase in the exchange surface. Moreover, high velocities of the lfuid are obtained due to reduced tubes cr $\square$-sections, leading to high pressure losses and consequently to large pumping power consumption. Also, the lfow distribution in the heat exchanger may be dififcult to control and additi $\square \square$ pressure losses are generated.

So, miniaturization of thermal systems involves finding dedicated solutions to maintain acceptable pumping power of the fluid simultaneously with high heat transfer and mixing efficiency. One possible way to simultaneously disturb the boundary layers and to integrate the pumping function within the heat exchanger is to generate a dynamic deformation of the channel's wall. Indeed, the disturbance of the boundary layer can be achieved by using dynamic wall deformation.

Various solutions can be practically considered to generate such wall movement, as it is done in some micro pumps. For instance, the actuation of the heat exchanger's wall can be done like the actuation of a membrane in a discrete peristaltic pump [1-3].

Nakamura et al., [4] and Kumar et al., [5] investigated numerically the inlfuence of the wall oscillating deformation $\square$ on heat transfer characteristics in a two dimensional channel. Kumar et al. present heat transfers analysis for circular millimeter size tubes with static and moving sinusoidal corrugated walls. The heat transfer coefifcient for the moving wav $\square \square$ walls (standing waves) had a higher value for all frequencies compared to the static wall case (up to $35 \square 70 \%$ ). A sharp decrease in pressure drop (by a factor of 1.2 to 5) was also obtained at high amplitudes. Mainly, heat transfer and pressure drop values apparently changed erratically with wall frequency. No general trend of heat transfer and pressure drop values in respect to operating parameters was found.

Leal et al., [6] presented a numerical study to determine both fluid flow and thermal performances of a heat exchanger channel deformed by a progressive sinusoidal wave. It was demonstrated that the pumping function could be integrated in the heat exchanger allowing enhancing the heat transfer in a significant way. The flow rate and the heat transfer coefficient are function of both the travelling wave amplitude and frequency. It was proposed to control independently the value of the pressure difference and the value of the heat transfer coefficient. Thus, this technique involving a dynamic deformation of one of the channel wall may be considered for practical applications.

The aim of the present paper is to study the thermo-hydraulic performances of a mobile corrugated channel within a heat exchanger while taking into account realistic boundaries and geometry.

\section{Geometry of dynamic channel}

In order to study fluid flow and heat transfer characteristics in small-scale heat exchanger, a virtual prototype based on a flat and straight channel and further transformed in a corrugated channel has been developed as presented in Figure 1 (detailed in section 3). Such a dynamically deformed corrugated channel allows us gaining a better understanding of heat transfer and pumping performances that are influenced by several operating parameters.

In the studied configuration, the lower wall was fixed and subjected to a constant and uniform heat flux over the imprint of heating zone length (length, L of 50mm), facing the actuated zone on the upper wall. On the other hand, the upper wall has been dynamically deformed to define five sinusoidal periods along this length and was kept adiabatic like the sidewalls. The height of the channel, average distance between the two walls $(\delta)$ was $1 \mathrm{~mm}$ while the width $(\mathrm{W})$ of the channel was $50 \mathrm{~mm}$. The upper wall deformation is progressively damped in inlet and outlet zones. In order to avoid numerical bias induced by reverse flow effect across boundaries, the lengths of inlet and outlet sections (reservoir zones) that were static in nature have been chosen sufficiently long $(50 \mathrm{~mm})$ to ensure a hydro-dynamically established flow at the entrance of the heated zone and to minimize boundary constraint at the exit of the actuated zone. Furthermore, a no-slip condition was imposed on all the physical walls.

The influence of amplitude (A) and imposed pressure difference at outlet-inlet of the exchanger $\left(\Delta \mathrm{P}_{\mathrm{s}}\right)$ for a given frequency $\left(f_{r}\right)$ and wavelength $(\lambda)$ on thermo-hydraulic performances is studied. 3-D numerical simulations have been carried out using the commercial software StarCCM+. This software allows modelling fluid flow and heat transfer in dynamically deformed structures. The lfow was considered tansient, three-dimensional and laminar. The working fluid was liquid water (at $27^{\circ} \mathrm{C}$ ). All its thermo-physical properties were supposed constant in the following systematic studies. Moreover, impact of variations in thermo-physical properties was studied for some configurations. The imposed heat power $(\Gamma)$ on the imprint (heated zone) was $125 \mathrm{~W}$. Parametric studies were carried out for the cases where the wave amplitude varies from $60 \%$ up to $98 \%$ of the channel height $(\delta)$ with an imposed frequency $\left(\mathrm{f}_{\mathrm{r}}\right)$ of $50 \mathrm{~Hz}$. The average height of the channel, i.e. the mean height of the membrane $(\delta)$ is kept constant. The change in amplitude thus changes the extremal distance between membrane 
and heated wall. In order to avoid effects due to inlet and outlet zone characteristics the results presented here focuses on the "active zone" namely between $\mathrm{P}_{1}$ and $\mathrm{P}_{2}$ sections (see Figure 1). All representative thermo-hydraulic quantities were calculated on this zone.
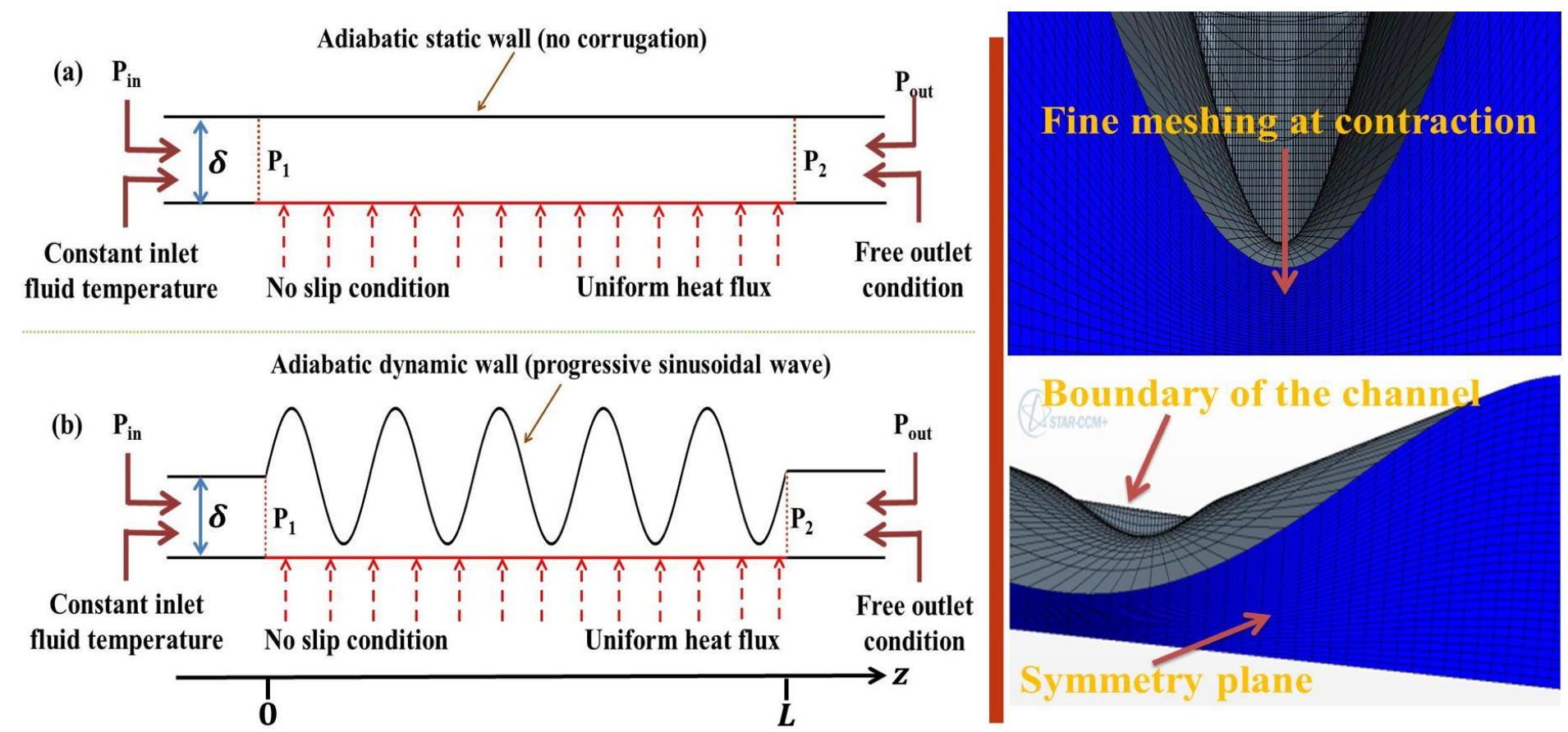

Fig. 1: Left: 2-D schema of (a) flat channel where the upper wall is straight and static, (b) corrugated channel where the upper wall is dynamically deformed. The lower wall is uniformly heated. The upper walls (straight and deformed) were kept adiabatic. A pressure difference $\left(\Delta P_{s}=P_{\text {out }}-P_{\text {in }}\right)$ is imposed between inlet and outlet sections of the channel. Right: Different meshing views.

\section{3-D numerical simulations}

The flow and heat transfer in unsteady laminar flow are solved by coupling the continuity, momentum and energy equations in 3-D. The geometry is symmetrical with respect to the vertical plane passing through the central axis of the channel. This leads to the reduction in number of mesh cells to optimize computation time by dividing the simulated configuration by two compared to its actual size. The power imposed on this half channel $(W=25 \mathrm{~mm})$ is thus $62.5 \mathrm{~W}$. Note that the fluid flow is considered in the z-direction while the width and height of the channel were considered in $x-$ and $y$-directions respectively. Images of the deformed corrugated wall and mesh views are presented in Figure 1.

\subsection{Management of dynamic corrugated wall}

Three functions have been used to impose the displacement of the mobile wall of the channel. The first describes the progressive sinusoidal wave, while the other two functions damp the movement in the vicinity of the lateral walls, and at the inlet and outlet of the actuated zone. The displacement of the upper wall (membrane) is defined by:

$$
y(z, t)=\delta+A \cdot\left(\Upsilon_{1} \cdot \Upsilon_{2}\right) \cdot \sin \left\{2 \pi\left(f_{r} t+\omega z\right)\right\}
$$

where, $\delta$ is the average height of the channel, $A$ is the amplitude of the wave, $\Upsilon_{1}$ and $\Upsilon_{2}$ are two threshold functions that takes into account the different membrane deformation effects in the vicinity of the boundaries of the actuated zone, $\mathrm{f}_{\mathrm{r}}$ is the frequency, $t$ is the time and $\omega$ is the number of waves per unit length respectively.

The two threshold functions are defined as:

For lateral damping: 


$$
\text { if } x>0.0015 m: \Upsilon_{1}=1 \text {; else }: \Upsilon_{1}=\left(\frac{x}{0.0015}\right)^{3}
$$

Similarly, for longitudinal damping:

$$
\begin{aligned}
& \text { if } z>0.05 m \text { and if } z \leq 0.10 m: \Upsilon_{2}=1 \\
& \text { if } z>0.10 m: \Upsilon_{2}=\left(1-\frac{z-0.10}{0.05}\right)^{4} \\
& \text { if } z<0.05 m: \Upsilon_{2}=\left(\frac{z}{0.05}\right)^{4}
\end{aligned}
$$

\subsection{Thermal parameters}

All thermo-hydraulic performances were calculated between $P_{1}$ and $P_{2}$ sections. The local heat transfer coefficient $\left(h_{\lambda}\right)$ is determined from the temporal averages of the fluid $\left(\bar{T}_{m f, \lambda}\right)$ and wall $\left(\bar{T}_{w, \lambda}\right)$ temperatures over a period, $\tau\left(\tau=1 / f_{r}\right)$ :

$$
\begin{gathered}
\bar{T}_{w, \lambda}(z)=\frac{1}{\tau W} \int_{t}^{t+\tau} \int_{x=0}^{W} T_{w}(x, z, t) d x d t \\
\bar{T}_{m f, \lambda}(z)=\frac{1}{\tau \delta W} \int_{t}^{t+\tau} \int_{y=0}^{y=\delta} \int_{x=0}^{x=W} T_{m f}(x, y, z, t) d x d y d t
\end{gathered}
$$

The mean heat transfer coefficient along $z$-direction is defined as:

$$
h_{\lambda}(z)=\frac{q(z)}{\left(\bar{T}_{w, \lambda}(z)-\bar{T}_{m f, \lambda}(z)\right)}
$$

where, $q$ is the heat flux imposed on the bottom wall.

In the following, the global heat transfer coefficient $(\langle h\rangle)$ across the channel is then defined as:

$$
\langle h\rangle=\frac{\Gamma}{S\left(\left\langle\bar{T}_{w}\right\rangle-\left\langle\bar{T}_{m f}\right\rangle\right)}
$$

where, $\Gamma$ is the power imposed on the heated surface and $S$ is the lateral surface area where flux is imposed.

\subsection{Mesh convergence criteria}

Mesh convergence criteria was performed on straight and flat channel (see Table 1). Based on these observations and the $\%$ difference in the global properties and to maintain a safety margin on the accuracy of the results, mesh size has been chosen that corresponds to 245752 cells to perform the systematic studies.

The same meshing criteria were applied for the dynamic wall case. The calculations were carried out until a periodic stationary regime is reached. This latter point is checked by comparing global heat transfer and flow characteristics: calculations are performed until the values of heat transfer coefficient between two consecutive time periods differ by less than $1 \%$. Consequently, an additional time period is added to extract all instantaneous and time-averaged values of all physical quantities. The chosen time step is small enough to allow an accurate description of the movement of the dynamic wall and all the thermo-hydraulic quantities. In the present work, 50 time steps per period are used.

\section{Systematic study}

Systematic thermo-hydraulic studies were first performed on flat and straight channel for the validation purpose and further extended to dynamic heat exchanger case. The impact of variable thermo-physical properties of fluid on global thermo-hydraulic properties was also investigated. It was found out that the heat transfer coefficients are slightly different 
(about 5\%) depending on constant and variable thermo-physical properties of water for the static and straight channel. On the other hand, heat transfer coefficient is slightly increased (variation in $\langle\mathrm{h}\rangle \approx 1 \%$ for $\mathrm{A}<90 \%$ ) and decreased (variation in $\langle\mathrm{h}\rangle<3 \%$ for $\mathrm{A}>90 \%$ ) compared to values obtained using constant properties of fluid for different amplitudes in using variable temperature dependent fluid properties. These tests were performed for negative pressure difference of $50 \mathrm{~Pa}\left(\Delta \mathrm{P}_{\mathrm{s}}<\right.$ 0 ). As the global thermo-hydraulic properties were not influenced by the present working thermal domain, systematic studies were further carried out using constant fluid properties.

Table 1: Global flow and heat transfer properties for different number of cells for flat and straight channel case (grey colour case chosen to carry out systematic studies while orange colour case shows loss in accuracy).

\begin{tabular}{cccc}
\hline Mass flow rate $(\mathrm{kg} / \mathrm{s})$ & Number of cells & Wall Temperature $(K)$ & Heat transfer coefficient $\left(W \cdot \mathrm{m}^{-2} \cdot \mathrm{K}^{-1}\right)$ \\
\hline & 5240 & 322.71 & 2722 \\
\multirow{2}{*}{$1.0 \mathrm{E}-02$} & 66900 & 322.79 & 2715 \\
& 245752 & 322.81 & 2714 \\
& 485368 & 322.82 & 2714 \\
\hline & 3782072 & 322.63 & 2734 \\
\hline & 5240 & 317.72 & 3305 \\
\hline & 66900 & 317.83 & 3290 \\
& 245752 & 317.85 & 3288 \\
& 485368 & 317.86 & 3287 \\
\hline
\end{tabular}

\subsection{Straight channel: Reference case validation}

Lee et al., [7] performed experiments with rectangular ducts heated on one of the longest sides by a constant imposed flux while other sides were kept adiabatic. These authors compared their results against different numerical (e.g. Wilbulswas [8]) and analytical (e.g. Phillips [9]) data and observed a good agreement (deviation varying from $2.4 \%$ to $8.1 \%$ ).

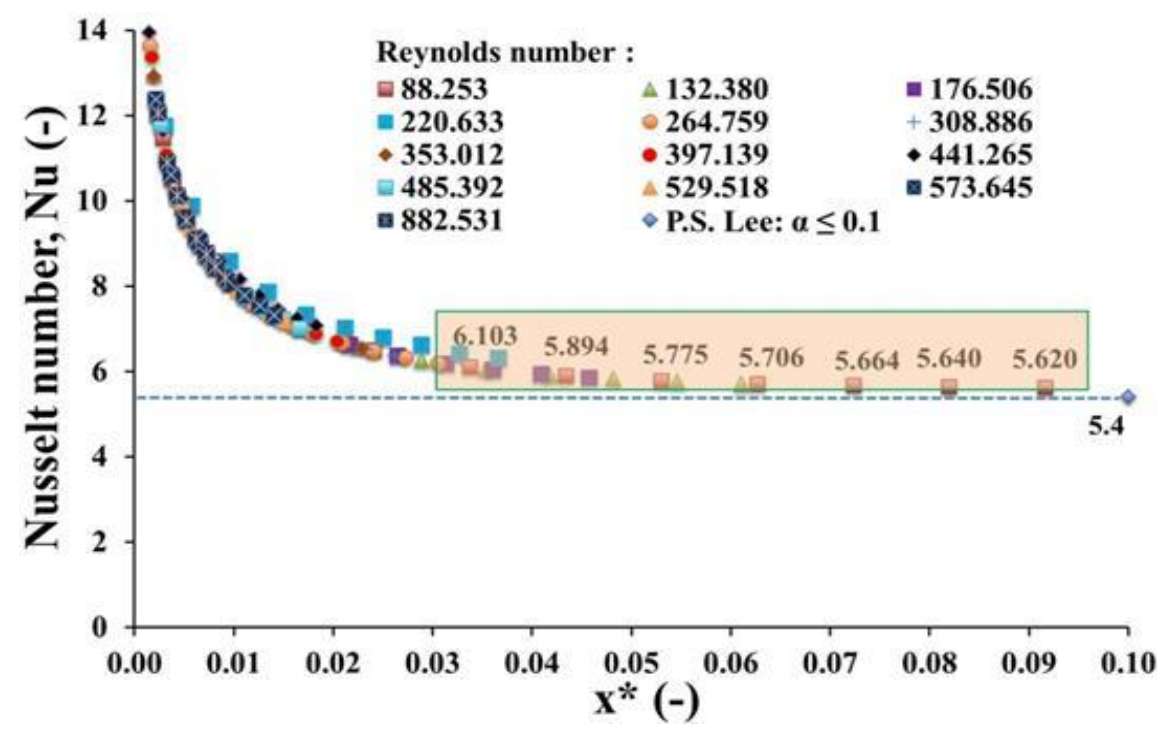

Fig. 2: Evolution of Nusselt number along $x^{*}$ for different Reynolds numbers in a rectangular channel heated by a constant heat flux on one side. Analytical results from P.S. Lee [7] are given for an aspect ratio: $\alpha \leq 0.1$. The data points in the green box represent the Nusselt numbers at various positions at different Reynolds number. 
For the reference case (straight channel) validation, current numerical data obtained using constant fluid properties at mean fluid temperature were validated against experimental data of Lee et al., [7] for the aspect ratio $\alpha \leq 0.1$ in terms of Nusselt number $(\mathrm{Nu})$ at a given dimensionless thermal axial distance $\left(\mathrm{x}^{*}\right)$ as presented in Figure 2. Note that, the aspect ratio of our straight channel is $\alpha=0.02$. The difference between current numerical results and experimental data of Lee et al., [7] when $\alpha \leq 0.1$ may be due to geometric differences of the working domain as well as temperature dependent fluid properties. This excellent validation of heat transfer and mass flow characteristics for the straight channel allows us to extend and explore the thermo-hydraulic performances of dynamic wall corrugated channel in the following section as a major objective of the current work.

\subsection{Dynamic corrugated channel}

The global thermo-hydraulic characteristics of mass flow rate, heat transfer as functions of negative pressure differences between exit and entrance sections of the dynamic corrugated channel $\left(\Delta \mathrm{P}_{\mathrm{s}}<0\right)$ of the studied system are presented in the following sections.

\subsubsection{Local Analysis}

Local analysis is presented for a dynamic heat exchanger when $\mathrm{A}=89 \%$ and $\Delta \mathrm{P}_{\mathrm{s}}=-10000 \mathrm{~Pa}$ at a given instantaneous time in Figure 3. There is an increase in pressure in the actuated zone (dynamic progressive wall) contrary to what is observed in classical flat and straight channel. Such an increase in pressure in the actuated region helps in increasing high mass flow rate, which in turn results in high heat transfer.
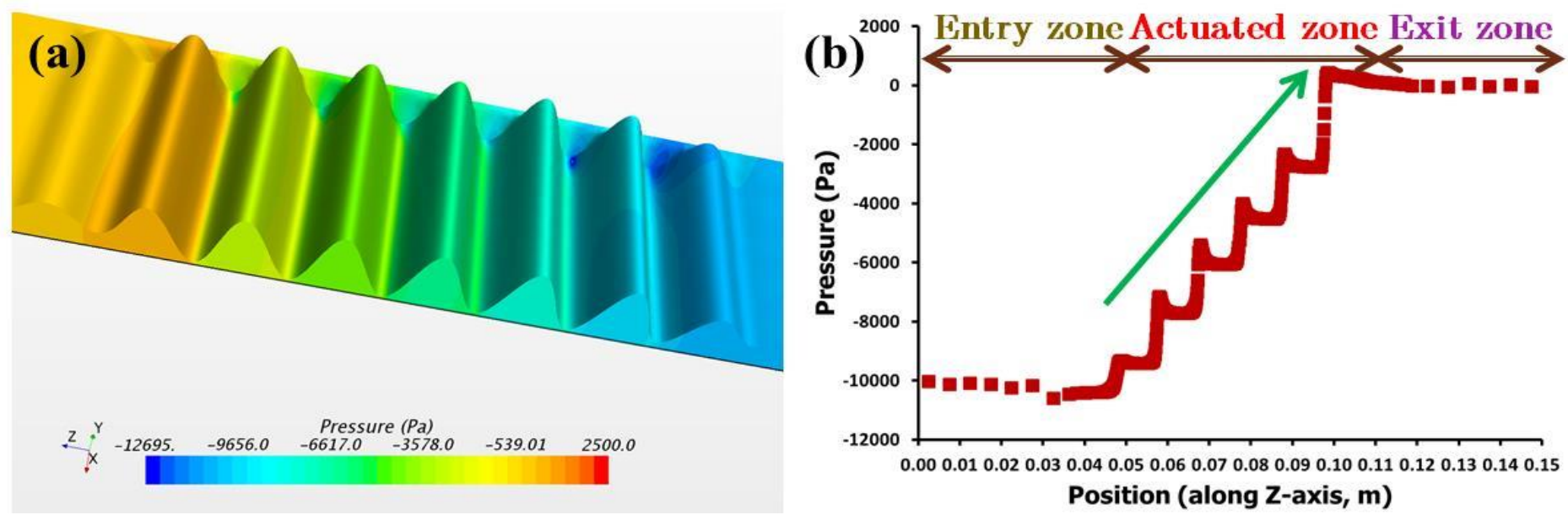

Fig. 3: (a) Instantaneous pressure field (b) Pressure profile along the channel length in the dynamic heat exchanger. Stair like profile in actuated zone: Pressure increases in each sinusoidal pocket.

\subsubsection{Global thermo-hydraulic performance}

Conventional corrugated channels (heat exchangers) need an external pump for the fluid to flow in the inlet-outlet direction of the channel because $\Delta \mathrm{P}_{\mathrm{s}}=\left(\mathrm{P}_{\text {out }}-\mathrm{P}_{\text {in }}\right)<0$. Without external pump, the channel could have a larger upstream pressure that would cause a flow in the reverse direction, so the channel may act as a brake to the flow. In the present study, the objective is to overcome such pressure loads and obstruction to the fluid flow using dynamic corrugated channel to compensate the pressure drop due to friction within the exchanger as well as the additional pressure drops due to the rest of the hydraulic circuit. Thermal-hydraulic characteristics in terms of mass flow and heat transfer coefficient are presented as a function of the imposed negative pressure difference between the outlet-inlet of the channel in Figure 4.

It is noted that for relatively small negative pressure differences $\left(-1000 \mathrm{~Pa}<\Delta \mathrm{P}_{\mathrm{s}}<-50 \mathrm{~Pa}\right)$ in the heat exchanger, the mass flow rate and heat transfer coefficient follows the same tendency as shown in the works of Leal et al., [6]. This suggests that the exchanger generates high mass flow rate for high amplitudes and thus, high heat transfer coefficient was obtained. For a given frequency $\left(\mathrm{f}_{\mathrm{r}}=50 \mathrm{~Hz}\right.$, in the present study), the dynamic wall drives the fluid in the same direction that the movement of sine waves. At low amplitudes, the wall pushes the fluid through simple moving contact. On the other hand, at high amplitudes (A $>85 \%$ ), fluid pockets are formed between each sine wave, which makes the flow reaching the value of 
the maximum flow rate. The heat transfer coefficient increases for high amplitudes because of the recirculation and acceleration of fluid exert in and between the pockets. Moreover, it is coupled to local pressures mainly located at the level lowest of the sine wave (minimum thickness) increasing transfer with imposed heat flux at the wall.

Higher value of negative pressure difference transforms into pressure drop and become more dominant with increasing amplitude. This leads the heat exchanger to act as a brake for the fluid flow. However, the heat transfer coefficient still attains a high value even for lower mass flow rate at higher negative pressure difference. On the other hand, it can be observed that when the amplitude increases $(A>90 \%)$, the mass flow rate and heat transfer coefficient tend to reach the same values even for lower pressure differences.
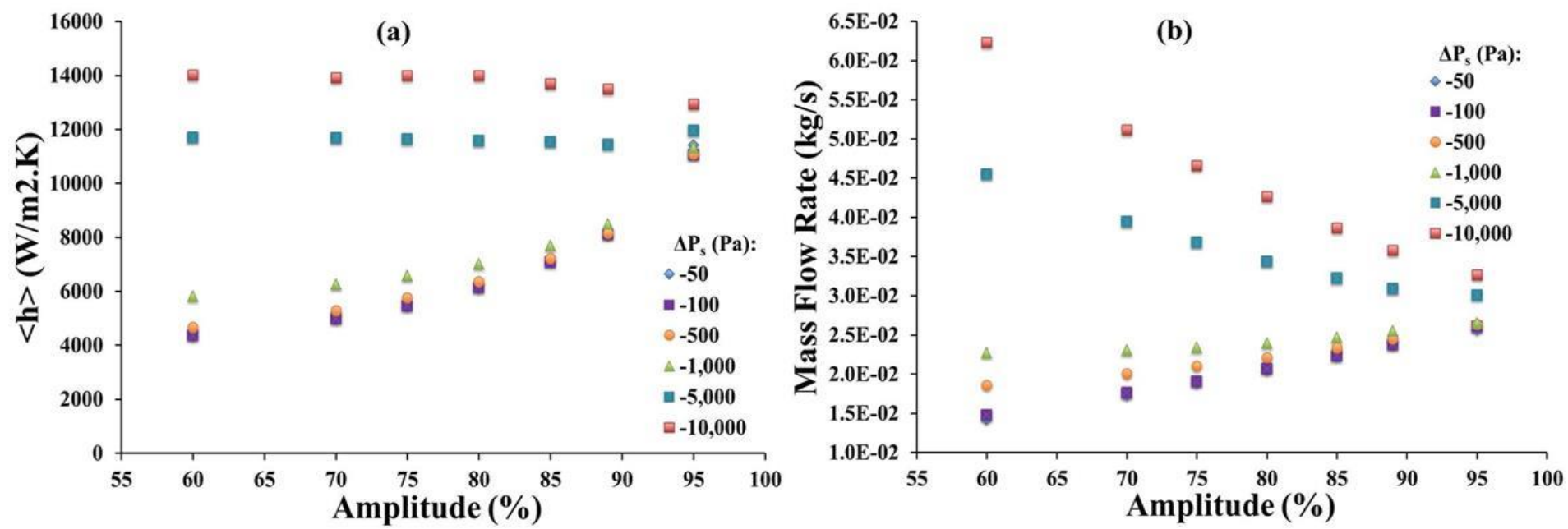

Fig. 4: (a) Global heat transfer coefficient $(\langle\mathrm{h}\rangle)$ as a function of amplitude for different $\Delta \mathrm{P}_{\mathrm{s}}<0$, (b) Mass flow rate as a function of amplitude for different $\Delta \mathrm{P}_{\mathrm{S}}<0$.

When pressure at the inlet is very high as compared to the outlet of the exchanger which connects directly to a prescribed flow rate, it limits the performance of the heat exchanger. Such a heat exchanger slows down greatly the fluid flow and thus, becomes 'ill-performed' because it requires more energy to obtain a dynamic corrugated wall to achieve a better performance.

\section{Merit factor}

Many researchers use the Colburn factor $(j)$ and friction factor $(f)$ to characterize heat transfer and pressure drop respectively. A surface having a higher $j / f$ factor is "good" because it requires lower free lfow area and hence a lower $\square$ frontal area for heat exchanger. The performance of heat exchangers can be evaluated as follows:

$$
\frac{j}{f}=\left(\frac{\langle N u\rangle}{\langle\operatorname{Re}\rangle \cdot \operatorname{Pr}^{\frac{1}{3}}}\right) /\left(\frac{\left\|\Delta P_{S}\right\| \cdot D_{h}}{\frac{1}{2} \rho\left\langle\|\vec{u}\|^{2}\right\rangle}\right)
$$

where, $\left\|\Delta \mathrm{P}_{\mathrm{S}}\right\|$ is absolute value of pressure drop.

Based on heat transfer obtained for negative pressure differences $\left(\Delta P_{S}<0\right)$, the performance of dynamically deformed corrugated channel for different amplitudes is presented in Figure 5. For a given pressure drop $\left(\Delta P_{S}=-50 \mathrm{~Pa}\right)$, it can be observed that highest performance of mobile heat exchanger can be achieved at high amplitudes. Compared to straight and flat channel, the performance is significant (see Figure 5a). On the other hand, the performance of the proposed system starts to decrease when pressure drop is quite high $\left(\Delta P_{S}=-10000 \mathrm{~Pa}\right)$ irrespective of the amplitude (see Figure 5b). It is quasiidentical what is observed in flat and straight channel. Moreover, when the pressure drop is moderate $\left(-5000 \mathrm{~Pa}<\Delta P_{S}<-\right.$ $1000 \mathrm{~Pa})$, the performance improves only at high amplitudes $(A>90 \%)$. Contrary to high-pressure drops, the performance amplifies to a very high extent when the pressure drop is low $\left(-100 \mathrm{~Pa}<\Delta P_{S}<-50 \mathrm{~Pa}\right)$ and at high amplitudes. 

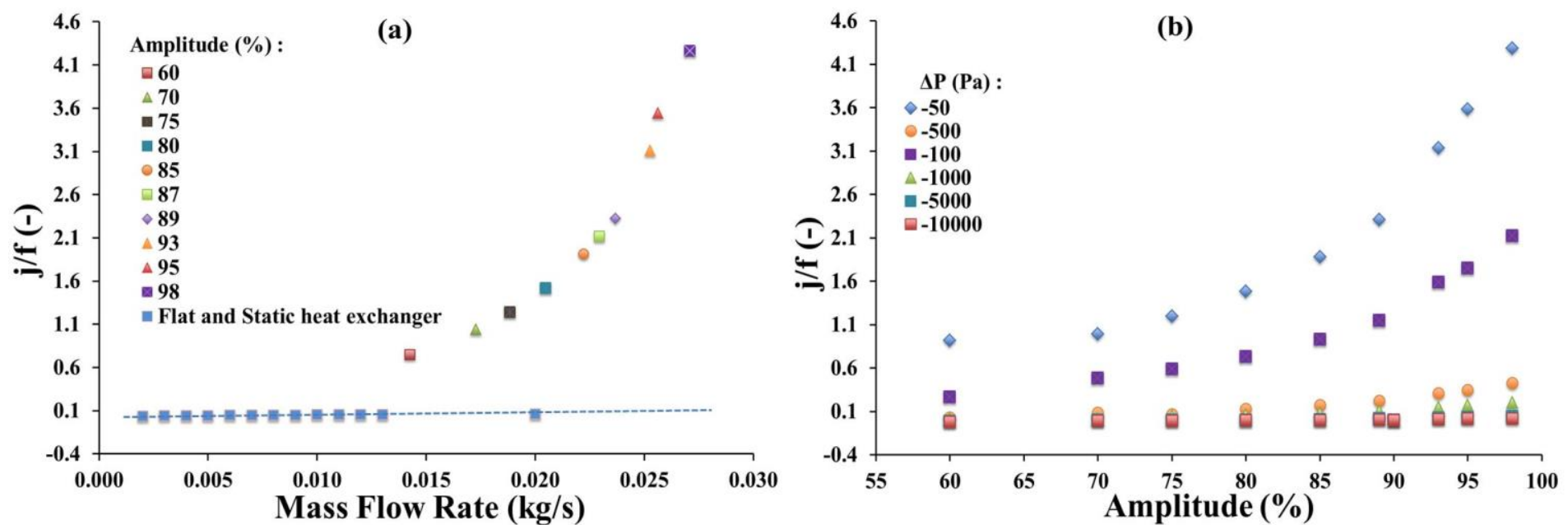

Fig. 5: (a) $j / f$ as a function of mass flow rate for different amplitudes at $\Delta P_{S}=-50 \mathrm{~Pa}$, (b) $\mathrm{j} / \mathrm{f}$ as a function of amplitudes for different $\Delta P_{S}$.

\section{Conclusion}

An innovative solution is proposed for the cooling of electronic devices that consists in dynamically deforming one of the walls of heat exchanger at millimetre scale within the channel. Thermo-hydraulic characteristics were investigated in liquid single-phase lfow and are found to be functions of amplitude and pressure drop. Heat transfer rate as well as mass flow rate follows the same increasing pattern at low amplitudes. On the other hand, at high amplitude, the mass flow rate possesses a decreasing behaviour while heat transfer rate doesn't vary significantly $\square$

A comprehensive characterization of dynamic heat exchanger was performed based on merit factor $(j / f)$. For the identical pressure drop conditions, the performance is increased by a factor of 10-46 for dynamically corrugated heat exchanger as compared to flat and straight channel. The proposed system's performance amplifies strongly at high amplitude and at low pressure drop. Thus, increase in the overall performance of the system and substantial gain in terms of integration can be achieved.

\section{Acknowledgements}

The authors would like to thank the financial support from FUI obtained in the framework of CANOPÉE (Contrôle Actif d'un draiN à calOries pour l'Electronique Embarquée) project.

\section{References}

[1] N.-T. Nguyen and X. Huang, "Miniature valveless pumps based on printed circuit board technique," Sensors and Actuators A: Physical, vol. 88, no. 2, pp. 104-111, 2001.

[2] M. Shen, L. Dovat, and M. A. M. Gijs, "Magnetic active-valve micropump actuated by a rotating magnetic assembly," Sensors and Actuators B: Chemical, vol. 154, no. 1, pp. 52-58, 2011.

[3] J. J. Allen and. J. Smits, "Energy harvesting EEL,” J. Fluids Struct, vol. 15, no. 3-4, pp. 629-640, 2001.

[4] M. Nakamura, T. Nakamura, and T. Tanaka, "A computational study of viscous flow in a transversely oscillating channel," JSME Int. J. Ser. C Mech. Syst. Mach. Elem. Manuf., vol. 43, no. 4, pp. 837-844, 2000.

[5] P. Kumar, F. Topin, M. Miscevic, P. Lavieille, and L. Tadrist, "Heat transfer enhancement in short corrugated minitubes," Numerical heat and mass transfer in porous media, Adv. Struct. Mater., vol. 27, pp. 181-208, 2012.

[6] L. Leal, F. Topin, P. Lavieille, L. Tadrist, and M. Miscevic, "Simultaneous integration, control and enhancement of both lfuid lfow and heat transfer in small scale heat exchangers: A numerical study," Int. Commun. Heat Mass., vol. 49, pp. 36-40, 2013.

[7] P. S. Lee, S. V. Garimella, and D. Liu, "Investigation of heat transfer in rectangular microchannels," Int. J. Heat Mass Transf, vol. 48, no. 9, pp. 1688-1704, 2005.

[8] P. Wibulswas, "Laminar-flow heat-transfer in non-circular ducts," Doctoral dissertation, University of London, 1966.

[9] R. J. Phillips, “Microchannel heat sinks,” Doctoral dissertation, Massachusetts Institute of Technology, 1987. 